\title{
Identifying Domestic Violence (DV) and sexual assault (SA) presentations and referral pathways at John Hunter Hospital Emergency Department: comparative analysis of DV and SA cases
}

Nafiseh Ghafournia ( $\sim$ Nafiseh.Ghafournia@health.nsw.gov.au )

NSW Health, Hunter New England Local health District, Multicultural Health

\section{Research}

Keywords: Domestic Violence, Sexual assault, Emergency Department, Domestic Violence screening, recurrent presentations

Posted Date: July 12th, 2021

DOI: https://doi.org/10.21203/rs.3.rs-665031/v1

License: (9) This work is licensed under a Creative Commons Attribution 4.0 International License. Read Full License 
Identifying Domestic Violence (DV) and sexual assault (SA) presentations and referral pathways at John Hunter Hospital Emergency Department: comparative analysis of DV and SA cases

Author: Nafiseh Ghafournia

Institution: NSW Health, Hunter New England Local health District, Multicultural Health

Address: Multicultural Health Service, Level 2 Harker Building Longworth Avenue Wallsend (Po Box 119) NSW 2287, Australia

Email: Nafiseh.Ghafournia@health.nsw.gov.au 


\begin{abstract}
Background: There are a number of negative and often long-term mental and physical health consequences of Domestic violence (DV) and Sexual assault (SA). Women experiencing DV and SA visit their health care professional more frequently than women not experiencing these abuses. Health care professionals in any health care settings can play crucial roles in identifying, managing and preventing DV and SA. In particular, those who work in emergency departments (EDs) are in a unique position to identify patients and initiate early interventions as they are often the first point of contact to access help.
\end{abstract}

Methods: This is a retrospective observational cross-sectional study. Data was extracted from the site ED electronic information system (ipm) for all eligible females (alleged assault) who presented within the study period. Statistical analyses were programmed using SAS v9.4 (SAS Institute, Cary, North Carolina, USA). All data were checked for completeness and discrepancies before analysis. Descriptive statistics are reported for all relevant variables. The Hosmer-Lemeshow goodness of fit test was used to determine the adequacy of fit between the final model and the data.

Results: Study findings indicate the high level of mental health issues among women presenting with both DV and SA. More than half of the victims had recurrent presentations to ED. The majority of victims were referred to the related support services within hospital or external services. Most of injuries in the sample were sever physical injuries.

Conclusions: As the first point of contact, ED health professionals have a crucial role to identify and respond to SA and DV cases. If identified early, the recurrent admissions can be prevented and any early intervention can have a positive benefit for the longer-term health of the DV and SA victims. It can also save health care system spending. The development of a DV/SA flowchart for identification of these cases in ED as well as a clear referral pathway and ideally mandatory DV routine screening at EDs in all hospitals would be some practical strategies for achieving early intervention.

Keywords: Domestic Violence, Sexual assault, Emergency Department, Domestic Violence screening, recurrent presentations 


\section{Background}

Domestic violence (DV) is a violation of human rights that disproportionately affects women. This is a serious, but preventable public health problem that is common worldwide. It is estimated that globally one woman in every three (35\%) has experienced physical and/or sexual violence at some point in their lives (WHO, 2016). The 2016 Australian Bureau of Statistics Personal Safety Survey (ABS, 2017) shows that one in 6 Australian women has been subjected, since the age of 15 , to physical and/or sexual violence by a current or previous cohabiting partner. Family, domestic and sexual violence happens repeatedly-more than half $(54 \%)$ of the women who have experienced current partner violence, experience more than one violent incident (ABS, 2017). One woman a week is killed by a current or former partner in Australia (Bryant and Bricknell, 2017). Furthermore, sexual assault can occur in the context of domestic and family violence (up to 40\%) (NSW Government, 2019). It can be a tactic of DV and should not be considered a separate phenomenon. Victims of sexual violence are also less likely to seek help than victims of other kinds of domestic violence (2019). For victims, there are a number of negative and often long-term mental and physical health consequences of different forms of domestic violence. Long-term physical health consequences of DV are intensely connected to long-term mental health consequences (Shen and Kusunoki, 2019).Victims of DV report higher rates of a range of health issues than non-victims (WHO, 2017; Ayre et al., 2016).Women experiencing DV visit their health care professional more frequently than women not experiencing DV (Gass et al., 2010; Rivara et al., 2007; Sprague et al., 2016). While there are important damaging effects on health for any kind of abuse (sexual, physical, psychological and emotional abuse, or neglect), health consequences may be worse for victims experiencing numerous forms of abuse co-occurring or combined over a lifetime (Laing, 2018; Taft, 2003; WHO, 2002).

Generally, health services provide a unique opportunity to identify women subjected to violence, provide them with appropriate care, connect them to other support services and, potentially, contribute to preventing future harm. For those who do seek professional help for violence, health-care providers are often women's first and most trusted point of professional contact (WHO, 2017). Health care professionals in any healthcare settings can play crucial roles in identifying, managing and preventing domestic violence (National Institute for Health and Care Excellence (NICE) 2014 ), but those who work in emergency departments 
(EDs) may regularly encounter people who have experienced IPV (Intimate Partner

Violence), who tend to present with injury or non-injury-related complaints (Houry et al., 2008). ED clinicians are therefore in a unique position to ask patients about DV and initiate early interventions (Ali et al., 2016), as most women are hesitant to disclose DV, particularly when they are not asked directly (Morse, et. al, 2012; Irwin and Waugh, 2001). Nurses and other ED healthcare professionals must be able to distinguish between injuries resulting from DV and those from other causes, and provide patient-centred, sensitive and empathetic care to the patients involved. The failure to recognise DV in ED fails women as they are also not provided with correct care and, consequently, best practice is not followed. The issues faced by women as a result of not being identified as a DV victim include continued risk, recurrent ED presentations which usually escalate over time. However, there is evidence that failure to recognise or respond to DV in ED is due in part to the ED clinician not seeing this as central to their role (Boursnell and Prosser, 2010).

\section{Domestic Violence Routine Screening}

Globally, DV Routine Screening has been introduced in some health systems for early identification of DV as well as to improve the quality of healthcare. The screening uses a standardised set of questions, irrespective of the presenting reason (García-Moreno et al., 2015; Hunter et al., 2017). The implementation of routine screening is a low-cost measure and provides an opportunity for identification and early intervention for women experiencing domestic violence (O’Doherty et.al, 2015; Spangaro and Ruane, 2014; WHO, 2013). Most states in Australia have introduced antenatal DV screening (Australian Institute of Health and Welfare, 2015), while New South Wales (NSW) also conducts screening in early childhood, mental health and substance abuse programmes (NSW Ministry of Health, 2016a). However, EDs do not systematically screen for IPV in Australia (Spangaro et al, 2020).

Domestic violence routine screening of eligible women was introduced in NSW in 2003 by the NSW Ministry of Health (2016a). The NSW Health Policy and Procedures for Identifying and Responding to Domestic Violence (PD2006_084) (NSW Department of Health, 2006) formalised this strategy and requires screening to be undertaken in four target services as part of routine assessment. In accordance with NSW Health policy, and guided by the privacy principles outlined in Schedule 1 of the Health Records and Information Privacy Act 2002 (NSW), the NSW Police force may be notified if the woman wishes and/or where there are 
concerns for the safety of the woman and/or her children. In all other cases where DV is identified but referral to the NSW Police or Department of Communities and Justice (DCJ) is not necessary, the referral pathway is guided by the woman's preferences and needs. Health workers will refer women to relevant health services or to services outside the health system. Nonetheless, the NSW Health Policy and Procedures for Identifying and Responding to Domestic Violence does not require routine screening in the ED (NSW Department of Health, 2006). This is concerning, as many victims of DV, ED is the first point of contact to access help (Dawson et.al, 2019). Prevalence of DV is higher among ED users than the general population and those in most other healthcare settings (Sprague et al., 2014, Spangaro et al., 2020).

It is important to note that rates of routine inquiry about exposure to DV are reported as very low in emergency settings, ranging from $2 \%$ to $13 \%$. A study by Webster et al. (2012) identified $12 \%$ of women attending ED did so as a result of an acute episode of DV, but less than one in seven of these women were asked about exposure to violence (Webster et.al, 2012). However, in line with The NSW Health Worker's Guide to Identifying and Responding to Domestic and Family Violence, (NSW health, 2019):

Irrespective of the health setting, NSW Health workers should respond to DFV, when a disclosure is made or DFV [Domestic and Family Violence] is suspected. Where indicators or inconsistent histories are present, staff should ask direct questions about the cause of injury or the dynamics within relationships... all NSW Health workers, however are required to ask direct questions about DFV where it is suspected (and it is safe to do so).

Furthermore, according to NSW Domestic and Family Violence Blueprint for reform: 20162021 (NSW Health Ministry, 2016b) and Integrated Prevention and Response to Violence, Abuse and Neglect Framework ( NSW Department of Health, 2019), population groups at higher risk of domestic and family violence are: Aboriginal people, people with disability, the lesbian, gay, bisexual, transgender, intersex and queer communities, people from culturally and linguistically diverse backgrounds, children, older women and people living in rural and remote areas. They need to be identified early and supported. Therefore, this study is significant as it will identify the number and characteristics of women presenting with DV 
and SA. This will assist health professionals with early intervention by improving referral pathways and, ultimately, result in a better quality of care for all.

\section{Method}

\section{Study aim}

This is a retrospective observational cross-sectional study. The aim of the study is to identify DV and SA presentations to the ED of a large metropolitan tertiary referral hospital in NSW, Australia for a period of one year. It provides a description of the characteristics of all females who presented to ED with alleged assault.

The following questions are addressed in this project:

- What is the prevalence of DV and SA presentations to ED?

- Are recurrent presentations identified in the data?

- What are the main characteristics of the presentations? For example, age, country of birth, being pregnant, having children, alcohol and drug abuse and mental health of the victim.

- What was the ED staff response to the cases? Were any referrals made to police, social workers, the child wellbeing unit or other support services?

- Is there any significant differences between DV and SA cases in terms of quantity and quality of referrals?

\section{Participants and setting}

John Hunter Hospital is the major trauma and teaching hospital for Hunter New England Health, NSW Health. It is located in Newcastle, NSW and is one of the busiest hospitals in NSW with between 18,000 to 22,000 ED presentations annually (Australian Institute of Health and Welfare, 2020).

The selection criteria is all females (no age limit) presenting with alleged assault to the ED at John Hunter Hospital (JHH) for the year 2018 (01/01/2018- 31/12/2018). There are a number of reasons for choosing this criteria: First, there are many indicators of DV such as cuts, bruises, miscarriage, headaches, body pains and many more, so collecting all possible DV cases based on these presentations is almost impossible. Second, we know that most cases of DV are not disclosed by the victims. Third, there is no standardised report for DV presentations at EDs. Therefore, after consulting with the ED team, it was decided that all female alleged assault 
cases be included in the study. It is noteworthy, that most of these cases are serious assaults with severe injuries.

\section{Data collection}

After obtaining research ethics approval from Hunter New England Human Research Ethics, data was extracted ( by one of ED staff member) from the site ED electronic information system (ipm) for all eligible females (alleged assault) who presented within the study period. Data collection included demographics such as: age, country of birth, aboriginality, having children, pregnancy, mental health, drug and alcohol abuse, triage (diagnosis), recurrent presentations and referral pathways. Following extracting the report, the data was de-identified by deleting indicators such as names, date of birth, address and Medical Record Number (MRN). In order to get a full picture of each case, the researcher looked into each case through DMR electronic system which contains scanned file for each patient presented at ED. The reason for this case by case investigation was to get more demographic information such as having children, pregnancy, abortion and termination which are not collected in the ipm report. In particular, recurrent presentations can only be identified by going through each patient's file to explore previous presentations. After identifying all demographic information needed, data were re -organised in an excel sheet. The de-identified data was sent to Hunter Medical Research Institute (HMRI) Clinical Research Design \& Statistics service for statistical analysis.

\section{Data analysis}

Statistical analyses were programmed using SAS v9.4 (SAS Institute, Cary, North Carolina, USA). All data were checked for completeness and discrepancies before analysis. Descriptive statistics are reported for all relevant variables, including frequencies and percentages for categorical variables, and means, standard deviations, medians, ranges and interquartile ranges for continuous variables. Logistic regression analyses were conducted to assess the associations with domestic violence versus sexual assault. Characteristics assessed included: age, aboriginality (ATSI), recurrent admission to John Hunter Hospital ED, mental health, pregnancy, referral to the relevant organisations, having children, alcohol/drug involvement. The variables mental health, having children and referral were re-categorised as yes/no indicator variables. The variable 'pregnancy' was also re-defined as yes for females who were pregnant at the time of incident and no for non-pregnant females and termination/miscarriage for females who had the experience of miscarriage/termination. The variable 'age' was categorised as $<18$ (child), 18-65 (adult) and 65+ (elderly). Characteristics identified at an alpha 
level of $5 \%$ on the Wald Chi-squared test in the logistic regression were considered statistically significantly associated with females who experienced domestic violence versus sexual assault. The Hosmer-Lemeshow goodness of fit test was used to determine the adequacy of fit between the final model and the data; with a $p$-value $>0.05$ on this test being considered an indication of an adequate fit. The area under the roc curve (AUC) was used to assess the discrimination ability of the final model, with a value of 0.7 or more considered acceptable. The influence of possible outliers was also assessed.

\section{Results}

There were 258 females identified as presenting for an alleged assault at ED, 97 for general alleged assault ${ }^{1}, 105$ for SA and 56 for due to DV. For the purpose of this study, only females who experienced DV and SA were included in the analyses. There is a possibility that some cases of alleged general assault might be domestic violence cases not disclosed by the victims. Table 1 describes the demographic characteristics of the study sample.

In the study, the majority of cases of SA (94\%) and DV (87\%) were Australian born. Country of birth for the non-Australian born cohort were mostly women born in New Zealand and the United Kingdom. The most common type of assault was SA (65\%). In the majority of SA cases there was no alcohol/drug involvement (72\%). Just over half of the victims had recurrent presentations to $\mathrm{ED}(51 \%)$ and $40 \%$ of cases had some kind of mental health issues. Fifteen percent identified as Aboriginal or Torres Strait Islander. The majority of women were neither pregnant nor had children, whilst $4.8 \%$ had at least one previous miscarriage or termination. On average, victims were aged 28 years of age (SD 14.4). Sixty six per cent of cases were between the ages of 18-65 years, while $32 \%$ were under the age of 18 . The mean age of SA cases is much lower than that of DV cases. The majority of victims were referred to the related support services $(88 \%)$. Most of the referrals were made to Hunter New England local sexual assault team and the Child Wellbeing Unit.

For the DV cases, almost half (48\%) had some sort of mental health issues. In the majority of cases there were no alcohol/drug involvement (61\%). More than half of the victims had recurrent admission to ED (52\%). Twenty one percent identified as Aboriginal or Torres Islander. Similar to SA cases, most of the women with experiences of DV were not pregnant

\footnotetext{
${ }^{1}$ Here, general alleged assault is defined as an assault inflicted on a female by a stranger in public settings.
} 
or did not have children. However, rates of miscarriage and termination were higher in SA cases than DV cases (8.9\% vs $4.8 \%)$. More than half of DV cases (52\%) had recurrent admission to ED. Ninety eight percent of DV cases were between the ages of 18-65 years, only $1.8 \%$ were under the age of 18 . The majority of victims were referred to the related support services (73\%). Most of the referrals were made to social workers, the Child Wellbeing Unit (when children were involved) or external organisations such as women's refuges, Department of Communities and Justice (DCJ), police or other DV support services. The most common injuries in the sample were strangulation, burning, poisoning, stabbing, breaking bones, and running over by car, as well as kidnapping and imprisonment for days, and deprivation of food, water and access to children. 


\begin{tabular}{|c|c|c|c|c|}
\hline \multirow[b]{2}{*}{ Variable } & \multicolumn{4}{|c|}{ Type of Assault } \\
\hline & & $\begin{array}{l}\text { Sexual } \\
\text { Assault } \\
(n=105)\end{array}$ & $\begin{array}{c}\text { Domestic } \\
\text { Violence } \\
(n=56)\end{array}$ & $\begin{array}{c}\text { Total } \\
(N=161)\end{array}$ \\
\hline \multirow[t]{2}{*}{ Mental health issue } & No & $63(60 \%)$ & $29(52 \%)$ & $92(57 \%)$ \\
\hline & Yes & $42(40 \%)$ & $27(48 \%)$ & $69(43 \%)$ \\
\hline \multirow[t]{2}{*}{ ATSI } & No & $89(85 \%)$ & $44(79 \%)$ & $133(83 \%)$ \\
\hline & Yes & $16(15 \%)$ & $12(21 \%)$ & $28(17 \%)$ \\
\hline \multirow{2}{*}{$\begin{array}{l}\text { Alcohol \& } \\
\text { involvement }\end{array}$} & No & $82(78 \%)$ & $34(61 \%)$ & $116(72 \%)$ \\
\hline & Yes & $23(22 \%)$ & $22(39 \%)$ & $45(28 \%)$ \\
\hline \multirow[t]{3}{*}{ Pregnancy } & No & $96(91 \%)$ & $42(75 \%)$ & $138(86 \%)$ \\
\hline & Pregnant & $4(3.8 \%)$ & $9(16 \%)$ & $13(8.1 \%)$ \\
\hline & $\begin{array}{l}\text { Miscarriage/Terminat } \\
\text { ion }\end{array}$ & $5(4.8 \%)$ & $5(8.9 \%)$ & $10(6.2 \%)$ \\
\hline \multirow[t]{2}{*}{ Having children } & No & $97(92 \%)$ & $38(68 \%)$ & $135(84 \%)$ \\
\hline & Yes & $8(7.6 \%)$ & $18(32 \%)$ & $26(16 \%)$ \\
\hline \multirow[t]{2}{*}{ Recurrent admission } & No & $51(49 \%)$ & $27(48 \%)$ & $78(48 \%)$ \\
\hline & Yes & $54(51 \%)$ & $29(52 \%)$ & $83(52 \%)$ \\
\hline \multirow[t]{2}{*}{ Referral } & No & $13(12 \%)$ & $15(27 \%)$ & $28(17 \%)$ \\
\hline & Yes & $92(88 \%)$ & $41(73 \%)$ & $133(83 \%)$ \\
\hline \multirow[t]{2}{*}{$\mathrm{COB}$} & Australia & $99(94 \%)$ & $49(87 \%)$ & $148(57 \%)$ \\
\hline & Other & $6(6 \%)$ & $7(13 \%)$ & $13(43 \%)$ \\
\hline \multirow[t]{3}{*}{ Age category } & $<18$ years & $34(32 \%)$ & $1(1.8 \%)$ & $35(22 \%)$ \\
\hline & 18-65 years & $69(66 \%)$ & $55(98 \%)$ & $124(77 \%)$ \\
\hline & $65+$ years & $2(1.9 \%)$ & & $2(1.2 \%)$ \\
\hline \multirow[t]{4}{*}{ AGE } & $\mathrm{n}$ & 105 & 56 & 161 \\
\hline & mean $(\mathrm{SD})$ & $\begin{array}{l}24.362 \\
(14.986)\end{array}$ & $34.875(10.241)$ & $28.019(14.396)$ \\
\hline & median $(\min , \max )$ & $21(0,85)$ & $34(16,57)$ & $27(0,85)$ \\
\hline & median (Q1, Q3) & $21(15,33)$ & $34(27,41.5)$ & $27(18,37)$ \\
\hline
\end{tabular}


Multivariable logistic regression analysis including all independent variables was fitted, with Maximum Likelihood Estimates and 95\% CIs presented. Table 2 details the adjusted odds ratios, 95\% CIs and Wald $p$-values for the characteristics investigated for association with the outcome: female experiencing DV or SA. A total of 161 females who had experienced either DV or SA were included in the final multivariable regression model; 56 of whom had experienced DV or SA. As shown in Table 2, age, having children and pregnancy were significantly associated with the outcome at the $p<0.05$ level, while controlling for the other characteristics. Aboriginal or Torres Strait Islander, alcohol and drug involvement, mental health and recurrent admission were not significantly associated with the outcome.

The odds of experiencing DV were increased by 1.06 times (OR: 1.06, 95\% CI: 1.03, 1.10) for each year of increase in age. Compared to females without children, those who had children had more than five times the odds (OR:5.47, 95\% CI: 1.93, 15.53) of experiencing DV. Compared to non-pregnant females, those who were pregnant had more than five times the odds (OR: 5.64, 95\% CI: 1.38, 23.01) of experiencing DV. The odds of experiencing DV in females who had had a miscarriage/termination during their pregnancy, were not significantly different to those for non-pregnant females (signified by the inclusion of 1 in the adjusted 95\% CI). 


\begin{tabular}{|c|c|c|c|}
\hline Characteristic & Category & $\begin{array}{c}\text { Adjusted OR } \\
(95 \% \mathrm{CI})\end{array}$ & $\begin{array}{c}\text { Adjuste } \\
d p- \\
\text { value }\end{array}$ \\
\hline Age & (per year) & $\begin{array}{l}1.06 \\
(1.03,1.10)\end{array}$ & 0.0001 \\
\hline $\begin{array}{l}\text { Aboriginal or Torres } \\
\text { Strait Islander }\end{array}$ & Yes & $\begin{array}{l}1.38 \\
(0.50,3.82)\end{array}$ & 0.5352 \\
\hline $\begin{array}{l}\text { Alcohol \& drug } \\
\text { involvement }\end{array}$ & Yes & $\begin{array}{l}2.01 \\
(0.84,4.79)\end{array}$ & 0.1146 \\
\hline Having children & Yes & $\begin{array}{l}5.47 \\
(1.93,15.53)\end{array}$ & 0.0014 \\
\hline Mental health issue & Yes & $\begin{array}{l}1.56 \\
(0.66,3.69)\end{array}$ & 0.3151 \\
\hline \multirow[t]{2}{*}{ Pregnancy } & $\begin{array}{l}\text { Miscarriage or } \\
\text { Termination }\end{array}$ & $\begin{array}{l}2.05 \\
(0.47,8.96)\end{array}$ & 0.3421 \\
\hline & Pregnant & $\begin{array}{l}5.64 \\
(1.38,23.01)\end{array}$ & 0.0160 \\
\hline Recurrent admission & No & $\begin{array}{l}1.90 \\
(0.73,4.92)\end{array}$ & 0.1861 \\
\hline
\end{tabular}

\section{Discussion}

The current study retrospectively reviewed data pertaining to female patients who presented to a busy metropolitan ED in NSW, Australia, as a result of alleged assault. The cases were divided into DV and SA. It is noteworthy that there were 258 female alleged assault presentations to only one ED over a 12 months period, particularly, when we know most women are hesitant to disclose DV, unless they are specifically asked (Morse, et al., 2012; Irwin and Waugh, 2001). Furthermore, many of these cases were serious physical injuries which could not possibly be overlooked by health professionals due to their severity. Therefore, this data does not show the true extent of the prevalence of DV and SA in EDs.

Study findings indicate the high level of mental health issues among women presenting with both DV and SA. This finding is in line with other research that provided evidence of the link between DV and anxiety and depressive disorder (Okuda et al., 2011; Suglia et al., 2011; Vos et al., 2006). The data clearly shows the pattern of referral made from the ED. Most of the SA and DV ED presentations were referred to the most relevant services. In particular, most of SA cases were referred to Hunter New England local sexual assault team and Child 
Wellbeing Unit child protection services and/or social workers. Although the referral rate is high and more than what is found in the current literature (Spangaro et al.; 2020), 27\% of DV cases were not referred. We know that these women are likely to have future ED presentations and, considering the severity of DV cases, this is a concerning finding.

The study had a number of limitations including its retrospective nature, small numbers and single site making it ungeneralizable to other sites. Another important limitation was that no data were collected regarding the actual effectiveness of ED staff responses and referrals. Furthermore, the research findings highlight areas where future research could be undertaken. An important finding from this pilot study is the strong correlation of both DV and SA with recurrent admissions to the same ED. As mentioned before, frequent health presentations to ED are indicators of DV. According to WHO (2013), these indicators include unexplained injury; chronic gastrointestinal, reproductive or genitourinary symptoms; repeated vaginal bleeding; traumatic injury, particularly if repeated and with vague or implausible explanations; and problems with the central nervous system including headaches, cognitive problems or hearing loss. If identified early, these frequent admissions can be prevented and any early intervention can have a positive benefit for the longer-term health of the DV and SA victims. It can also save health care system spending. Another key areas in need of further research include survey of SA and DV patients presented at ED; interviewing with health professionals including doctors, nurses and social workers through few focus groups. It would be ideal to capture the staff's views and perspectives on how to address the issue. Also, conducting research in different hospitals and different local health areas could form the basis of a comparative study.

Importantly, early detection and intervention are dependent on health care professionals recognising DV and SA so that response is early and appropriate. This can only be achieved if health care professions have adequate training in this area. This is an area for future research, to develop, implement and evaluate these tools. Also, development of a DV flowchart for identification of DV and SA cases in ED, as well as a clear referral pathway, in particular to social work team, would be one practical strategies for achieving early intervention. Ideally, DV routine screening should be mandated at EDs in all hospitals. Although, DV screening does not currently apply to EDs, according the NSW Health Worker's Guide to Identifying and Responding to Domestic and Family Violence (NSW Health, 2019), irrespective of the health setting, NSW Health workers should know how to 
respond to domestic and family violence when there is a disclosure of DV or suspicion of it occurring. In all these cases, all NSW health workers should ask direct questions about the cause of injury.

\section{Conclusion}

This study highlights the importance of gaining a more robust understanding of DV and SA prevalence and the characteristics of the women who present to our EDs having suffered DV or SA. This understanding is fundamental in facilitating appropriate prevention and early intervention to improve the health outcomes of these women. This is particularly relevant within ED settings, often the first point or sometimes last point of contact for many women who are victims of DV and SA. Whilst the specific results of this study are not generalizable, the findings will be of interest to other hospitals in different settings as they provide, for the first time, a description of DV presentations to ED, their characteristics, types of referral, staff responses, and impact on the future wellbeing of patients. What is clear from this study is the significant role of the health system in identifying and responding to the increasingly prevalent, damaging and costly problem of SA and DV. Despite the challenges of conducting research within this population, an emerging body of evidence points to the critical role of the health system in reducing the harms caused by SA and DV. In particular, the evidence points to investment in screening of selected vulnerable groups, training for first-line responders and expansion of interventions through an appropriate referral pathway and the essential role of social workers and enhancing their roles in the EDs. The long-term health costs arising from the epidemic of SA and DV mean that these are investments are likely to contribute to cost savings. 


\section{Declarations:}

\section{Ethical Approval and Consent to participate}

This study received Ethics approval from Hunter New England Health Human Research Ethics committee (Application ID: 2018/ETH00694).

\section{Consent for publication}

Not pplicable

\section{Availability of data and materials}

The datasets generated and analysed during the current study are not publicly available due [sensetivity of the cases and difficulty in obtaining consents from patients who attended ED in last 12 months priror to the study] but are available from the corresponding author on reasonable request

\section{Competing interests}

The author declares that there is no competing interests.

\section{Funding}

This research recieved funding from Hunter New England Health Research Translation Centre for the cost of statistical analysis.

\section{Authors' contributions}

Not applicable

\section{Acknowledgements}

I wish to acknowledge John Hunter Hospital (JHH) Emergency Department managers and staff and the social work team for their contribution to this project. Special thanks to Jody Jessop, JHH ED social worker, for her valuable support. I am particularly grateful for the assistance provided by Jeremy Pallas, JHH ED Nurse Educator, in collecting the ipm data. I would also like to acknowledge the support provided by Hunter Medical Research Institute (HMRI), in particular, the assistance provided by Doctor Chris Oldmeadow, Associate Director of Clinical Research Design and Statistics and Sima Ahmadi, the statistician who analysed the data. I would also like to express my very great appreciation to Ashley Young, Manager of Multicultural Health service for his valuable and constructive suggestions and support during the planning and development of this research study.

\section{Authors' information}

Nafiseh Ghafournia PhD

Affiliations:

1. Hunter New England Health, NSW Health, Australia

2. The university of Newcastle, NSW, Australia 


\section{References}

- Ali, P; McGarry, J; Dhingra, K. (2016). Identifying signs of intimate partner violence Emergency Nurse, 23(9), 25-29.

- Australian Bureau of Statistics, (2017). Personal Safety Survey 2016. ABS cat. no. 4906.0. Canberra: ABS.

- Australian Institute of Health and Welfare, (2015).Screening for Domestic Violence during Pregnancy: Options for Future Reporting in the National Perinatal Data Collection Information Paper. Canberra: Australian Government.

- Australian Institute of Health and Welfare, (2020). John Hunter Hospital, Emergency department, presentations.

- Ayre, J; Lum On, M; Webster, K; Gourley, M; Moon, L, (2016). Examination of the burden of disease of intimate partner violence against women in 2011: Final report (ANROWS Horizons, 06/2016). Sydney: ANROWS.

- Boursnell, M; Prosser, S, (2010). Increasing identification of domestic violence in emergency departments: A collaborative contribution to increasing the quality of practice of emergency nurses Contemporary Nurse, 35 (1), 35-46.

- Bryant W, Bricknell S, (2017). Homicide in Australia 2012-13 to 2013-14: National Homicide Monitoring Program Report. Canberra: Australian Institute of Criminology.

- Dawson, A. J; Rossiter, C; Doab, A; Romero, B; Fitzpatrick, L; Fry, M, (2019). The Emergency Department Response to Women Experiencing Intimate Partner Violence: Insights from Interviews with Clinicians in Australia, American academy Medicine, 26 ( 9), 1052-1062.

- García-Moreno C, Hegarty K, d'Oliveira A, Koziol-McLain J, Colombini M, Feder G, (2015). The health systems response to violence against women. Lancet, 385 (9977) $1567-1579$.

- Gass, JD; Stein DJ; Williams, DR; Seedat, S, (2010 0. Intimate partner violence, health behaviours, and chronic physical illness among South African women, South African Medical Journal, 100(9), 582-285.

- Hunter, T; Botfield, J. R ; Estoesta, J ; Markham, P; Robertson, S; McGeechan, K, (2017). Experience of domestic violence routine screening in Family Planning NSW clinics, Sexual Health, 14(2), 155-163.

- Houry, D; Kaslow, N; Kemball, R; et al, (2008). Does screening in the emergency department hurt or help victims of intimate partner violence? Annals of Emergency Medicine. 51(4), 433- 442.

- Irwin J; Waugh, F, (2001). Unless they're asked: Routine screening for domestic violence in NSW Health - an evaluation report of the pilot project, NSW Health.

- Laing, L, (2018). Broadening the frame: how ways of 'seeing' shape ways of 'doing' (Conference paper), Violence, Abuse and Neglect Clinical Forum Sydney: 23 July, 2018. 
- Morse, DS; Lafleur, R; Fogarty, CT; Mittal, M; Cerulli, C; (2012). "They told me to leave": how health care providers address intimate partner violence, Journal of the American Board of Family Medicine, 25(3), 333-342.

- National Institute for Health and Care Excellence, (2014). Domestic Violence and Abuse: How Social Care, Health Services and Those They Work with Can Respond Effectively. NICE, London. 26361313.

- NSW Ministry of Health, (2016a). Domestic violence Routine Screening November 2015 snapshot 13, Prevention and Response to Violence abuse and neglect Unit.

- NSW Ministry of Health, (2016b). NSW Domestic and Family Violence Blueprint for reform: 2016-2021: safer lives for Women, men and Children.

- NSW Department of Health, (2006). Policy and procedures for identifying and responding to domestic violence, Sydney: NSW Department of Health.

- NSW Department of Health, (2019). Integrated Prevention and Response to Violence, Abuse and Neglect Framework, PD2019_041.

- NSW Government, Hunter New England local Health District, (2019). The NSW Health Worker's Guide to Identifying and Responding to Domestic and Family Violence.

- NSW Ministry of Health, (2019). Prevention and Response to Violence, Abuse and Neglect. NSW Health Domestic Violence Screening and Response in NSW Emergency Department: a feasibility study, Government Relations Branch. Sydney.

- O’Doherty L, Hegarty K, Ramsay J, Davidson L, Feder G, Taft A, (2015). Screening women for intimate partner violence in healthcare settings. Cochrane Database Systemic Review, 7: CD007007.

- Okuda, M; Olfson, M; Hasin, D., Grant, B. F; Lin, K. H; Blanco, C, (2011), Mental health of victims of intimate partner violence: Results from a national epidemiologic survey. Psychiatric Services, 62 (8), 959-962.

- Rivara, FP; Anderson, ML; Fishman, P; Bonomi, AE; Reid, RJ,; Carrell, D; Thompson, RS, (2007). Healthcare utilization and costs for women with a history of intimate partner violence. American Journal of Preventative Medicine, 32 (2), 89-96.

- Shen, S., \& Kusunoki, Y, (2019). Intimate partner violence and psychological distress among emerging adult women: A bidirectional relationship. Journal of Women's Health, 28(8), 1060-1067, doi:10.1089/jwh.2018.7405.

- Spangaro J, Ruane J, 2014, Health interventions for family and domestic violence: a literature review. Sydney: University of New South Wales.

- Spangaro, J; Vajda, J; Klineberg, E; Lin, S; Griffiths, C; Saberi, E; Field, E; Miller, A; McNamara, L, (2020). Intimate partner violence screening and response in New South Wales emergency departments: A multi-site feasibility study, Emergency Medicine Australia, 10 (1111),1-21 doi: 10.1111/1742-6723.13452. 
- Sprague, S; Goslings, JC; Hogentoren, C; De Milliano, S; Simunovic, N; Madden, K, Bhandari, M, (2014). Prevalence of intimate Partner violence across medical and surgical health care settings: a systematic review. Violence against Women. 20(1), 118-136. doi: 10.1177/1077801213520574 PMID: 24476759

- $\quad$ Sprague, S; Slobogean, GP; Spurr, H; McKay, P; Scott, T; Arseneau, E, Memon,M; Bhandari, M; Swaminathan, A, (2016). A Scoping Review of Intimate Partner Violence Screening Programs for Health Care Professionals. PLoS ONE, 11(12), 1-17, e0168502. doi: 10.1371/journal.

- Taft, A, (2003). Promoting Women's Mental Health: The challenges of intimate/domestic violence against women. Sydney: Australian Domestic and Family Violence Clearinghouse, University of New South Wales.

- Vos, T; Astbury, J; Piers, L. S; Magnus; A; Heenan, M; Stanley, L; Webster, K, (2006). Measuring the impact of intimate partner violence on the health of women in Victoria, Australia. Bulletin of the World Health Organization, 84 (9),739-44.

- Webster S; Pedrosa, C; Lopez, V, (2012). Domestic violence against women: incidence and prevalence in an emergency department population. Journal of Nursing Education Practice, 2(4), 145-153.

- World Health Organization, (2002). World report on violence and health. Available: https://apps.who.int/iris/bitstream/handle/10665/42495/9241545615_eng.pdf;jsessionid= A63114C4F440F11EE8A04D45E401A185?sequence=1, Accessed 6 May 2019.

- World Health Organization, (2013). Responding to Intimate Partner Violence and Sexual Violence against Women: WHO Clinical and Policy Guidelines. Geneva, Available: https://apps.who.int/iris/bitstream/handle/10665/85240/9789241548595_eng.pdf;jsessioni

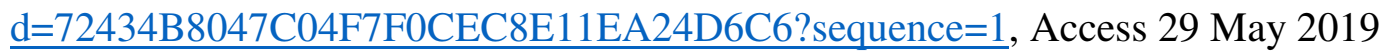

- World Health Organization (WHO), (2016). Violence against women: Intimate partner and sexual violence against women, Available: https://www.who.int/news-room/factsheets/detail/violence-against- Accessed 11 July 2019.

- World Health Organization (WHO), (2017). strengthening health systems to respond to women subjected to intimate partner violence or sexual violence: a manual for health managers, Geneva, Switzerland, Available at https://apps.who.int/iris/bitstream/handle/10665/259489/9789241513005eng.pdf?sequence=1, Accessed 21 March 2019. 
